\title{
Squid neurofilaments
}

\section{Phosphorylation and $\mathrm{Ca}^{2+}$-dependent proteolysis in situ}

\author{
Anthony BROWN and Peter A. M. EAGLES
}

Department of Biophysics, King's College (University of London), 26-29 Drury Lane, London WC2B 5RL, U.K.

\begin{abstract}
Three major polypeptides co-purify with neurofilaments from squid (Loligo forbesi) axoplasm: P60 (apparent $M_{\mathrm{r}}$ 60000), P200 (apparent $M_{\mathrm{r}}$ 200000) and Band 1 (apparent $M_{\mathrm{r}}$ 400000). Anti-IFA, a monoclonal antibody that recognizes an epitope common to all classes of intermediate filaments, binds to $\mathbf{P} 200$ and P60. When axoplasm is incubated with $\left[{ }^{32} \mathrm{P}\right] \mathrm{P}_{\mathrm{i}}$, the major phosphorylated polypeptides are $\mathrm{P} 200$ and Band 1. We have investigated $\mathrm{Ca}^{2+}$-dependent proteolysis of $\left[{ }^{32} \mathrm{P}\right]$ phosphorylated axoplasm in order to localize the major sites of phosphorylation and to probe the arrangement of the polypeptides in the filament. The proteinase preferentially cleaves P200 and Band 1, liberating the phosphorylated domains. Analysis of proteolysed filaments by electron microscopy and gel electrophoresis shows that most of P200 and Band 1 can be cleaved while still maintaining intact filaments. We suggest that P200 is initially cleaved within a single highly sensitive region, generating two major fragments called P100p (apparent $M_{\mathrm{r}} 100000$ ) and P110s (apparent $M_{\mathrm{r}}$ 110000). P100p contains the Anti-IFA epitope and co-sediments with filaments, whereas P110s is highly phosphorylated and does not sediment with filaments. Band 1 is cleaved to produce a soluble high- $M_{\mathrm{r}}$ fragment that is phosphorylated and that represents a major portion of the undigested component, whereas P60 is relatively resistant to limited proteolysis. Thus proteolysis appears to define two major filament domains: a conserved core that forms the backbone of the filament, and a highly phosphorylated peripheral region that is not essential for filament integrity.
\end{abstract}

\section{INTRODUCTION}

Neurofilaments from the giant axon of the squid Loligo appear to be composed of three distinct components: two major polypeptides of apparent $M_{\mathrm{r}}$ by SDS/polyacrylamide-gel electrophoresis of 200000 (P200) and 60000 (P60), and a high- $M_{\mathrm{r}}$ component (Band 1). The evidence for this comes from purification of neurofilaments by a variety of procedures, including cytochrome $c$ precipitation (Gilbert, 1976), discontinuous sucrose-gradient centrifugation and gel filtration (Lasek et al., 1979) and Millipore filtration (Roslansky et al., 1980). In these studies, $P 200$ and P60 are consistently found to be the major polypeptides that co-purify with the filament fraction. Co-purification of a third component, Band 1, has also been noted in a number of studies, especially those in which lower-percentage gels were used (Lasek et al., 1979; Eagles et al., 1980; Wais-Steider et al., 1983). Estimates of the apparent $M_{\mathrm{r}}$ of Band 1 by SDS/polyacrylamide-gel electrophoresis vary from $>400000$ (Pant et al., 1978) to 800000 (Eagles et al., 1980). This is probably due to its diffuse nature and low mobility, which may vary with the particular gel system used, rather than to an actual variation in molecular mass.

Neurofilaments from Loligo, as with neurofilaments from the fanworm Myxicola and from various mammalian sources, are substrates for two axoplasmic enzymic activities: phosphorylation and $\mathrm{Ca}^{2+}$-dependent proteolysis. In the case of squid, incubation of axoplasm with $\left[\gamma-{ }^{32} \mathrm{P}\right] \mathrm{ATP}$ results in phosphorylation of Band 1 and P200 by a specific endogenous $\mathrm{Mg}^{2+}$-dependent, $\mathrm{Ca}^{2+}$ independent and cyclic nucleotide-independent protein kinase (Pant et al., 1978, 1979; Eagles et al., 1980).

Neurofilaments can also be modified by an endogenous $\mathrm{Ca}^{2+}$-dependent proteolytic activity. Gilbert et al. (1975) have shown that incubation of axoplasm with $>0.5 \mathrm{~mm}$ ionized $\mathrm{Ca}^{2+}$ results in destruction of neurofilaments, as assayed by electron microscopy, apparently due to proteolysis of the neurofilament polypeptides. More recently, Pant \& Gainer (1980) have identified a soluble proteolytic activity from squid axoplasm that preferentially cleaves the P200 neurofilament polypeptide, with degradation proceeding via polypeptides of $M_{\mathrm{r}} 100000$ and 47000-50000.

Though the function of neurofilament phosphorylation and the physiological significance of $\mathrm{Ca}^{2+}$-dependent proteolysis are still matters of speculation, a study of these enzymic processes can provide valuable information on the structure and possible roles of this cytoskeletal component. In this paper we present results of studies on the phosphorylation and $\mathrm{Ca}^{2+}$-dependent proteolysis of squid neurofilaments. Limited proteolysis defines two major filament regions: a conserved core domain that forms the 'backbone' of the filament, and a highly phosphorylated peripheral domain that can be cleaved off without affecting filament integrity.

\section{METHODS}

Squid

Much of the work was carried out at the laboratory of the Marine Biological Association of the United Kingdom at Plymouth, Devon, U.K., between October and January. Squid (Loligo forbesi) were kept there in tanks of circulating seawater and survived for up to $48 \mathrm{~h}$ after capture. Squid that had been badly damaged during capture were decapitated and eviscerated, and the mantles were stored in ice-cold seawater; they were dissected within $2-6 \mathrm{~h}$ of death. The primary and 
occasionally secondary stellar giant axons were dissected from the mantles in cold seawater and fine-cleaned of associated nerve fibres over a length of about $1 \mathrm{~cm}$ at their proximal ends. The axoplasm was then extruded from the cleaned end, with care taken to avoid extracellular contamination.

\section{Phosphorylation and $\mathrm{Ca}^{2+}$-dependent proteolysis}

$\left[{ }^{32} \mathrm{P}\right] \mathrm{P}_{\mathrm{i}} \quad(1 \mathrm{mCi} / \mathrm{ml}$, carrier-free, Amersham International) was dried under vacuum and redissolved in buffer. Maximal phosphorylation of axoplasmic protein was achieved by incubating intact, freshly extruded, axoplasm in an equal volume of $\left[{ }^{32} \mathrm{P}\right] \mathrm{P}_{\mathrm{i}} / \mathrm{KGlu} /$ sucrose buffer (0.2 M-potassium glutamate $/ 0.6 \mathrm{M}$-sucrose $/ 5 \mathrm{~mm}$ $\mathrm{MgCl}_{2} / 0.1 \mathrm{~mm}$-sodium EGTA/0.01\% 2-mercaptoethanol, pH 7.2) at about $10^{\circ} \mathrm{C}$ for $12-18 \mathrm{~h}$. Typically, $1 \mu \mathrm{Ci}$ of $\left.{ }^{32} \mathrm{P}\right] \mathrm{P}_{i}$ was used per mg wet wt. of axoplasm. Proteolysis was initiated by addition of $\mathrm{CaCl}_{2}$ in $\mathrm{KGlu}$ /sucrose buffer to a final concentration of $10 \mathrm{~mm}$, and terminated by addition of the inhibitor tosyl-lysylchloromethane ('TLCK') to a final concentration of $10 \mathrm{~mm}$. The axoplasm was then homogenized on ice in $20 \mathrm{~mm}$ Tris $/ \mathrm{HCl}$ buffer, $\mathrm{pH} 7.2$, containing $0.1 \mathrm{M}-\mathrm{KCl}$ and $0.01 \%$ 2-mercaptoethanol to a final concentration of $40 \mathrm{mg}$ wet wt. of axoplasm $/ \mathrm{ml}$, and the homogenates were centrifuged at $120000 \mathrm{~g}\left(r_{\text {av }} .55 .7 \mathrm{~mm}\right)$ for $90 \mathrm{~min}$ at $5-10^{\circ} \mathrm{C}$ in order to separate filament (pellet) and soluble (supernatant) fractions for analysis by SDS/ polyacrylamide-gel electrophoresis.

\section{Enzymic dephosphorylation}

Axoplasm and alkaline phosphatase (type III, from Escherichia coli; Sigma Chemical Co.) were dialysed separately against dephosphorylation buffer (150 mM$\mathrm{NaCl} / 1 \mathrm{~mm}-\mathrm{ZnCl}_{2} / 50 \mathrm{~mm}$-Tris/HCl, $\mathrm{pH} \mathrm{10)}$. Dephosphorylation was then achieved by incubating axoplasm (concentration $1 \mathrm{mg}$ of protein $/ \mathrm{ml}$ ) with alkaline phosphatase at $37^{\circ} \mathrm{C}$ for $18 \mathrm{~h}$. Control incubations were carried out in the absence of enzyme, or in the presence of $0.5 \mathrm{M}$-sodium phosphate and $50 \mathrm{mM}$-EDTA to inhibit phosphatase activity.

\section{Electrophoresis}

Samples were analysed on 3-15\% polyacrylamide gels in the presence of SDS with a 3\% stacking gel and a discontinuous Tris/glycine buffer system, as described by Laemmli (1970). Gels were stained with Coomassie Brilliant Blue R-250 and photographed with an orange/green filter. $M_{\mathrm{r}}$ values in the range 13900-398000 were estimated by using glutaraldehyde-cross-linked phosphorylase $a$, rabbit muscle myosin, phosphorylase $a$, bovine serum albumin, rabbit muscle actin and lysozyme as standards. Densitometry of gels was performed on a Joyce-Loebl Chromoscan 3 densitometer at a wavelength of $626 \mathrm{~nm}$. For autoradiography, gels were exposed to Kodak X-Omat SX-ray film at $4{ }^{\circ} \mathrm{C}$.

\section{Immuno-blotting}

Gels were first pre-blotted to remove any surface protein by applying a nitrocellulose membrane (pore size $0.45 \mu \mathrm{m}$; Schleicher und Schüll), moistened in phosphatebuffered saline (137 mM-NaCl/3 mM-KCl/8 mM-Na ${ }_{2}^{-}$ $\mathrm{HPO}_{4} / 1.5 \mathrm{mM}-\mathrm{KH}_{2} \mathrm{PO}_{4}$ buffer, $\mathrm{pH}$ 7.2), to each side of the gel for a few minutes. This nitrocellulose was then discarded and the gel sandwiched between two fresh sheets of phosphate-buffered-saline-soaked membrane.

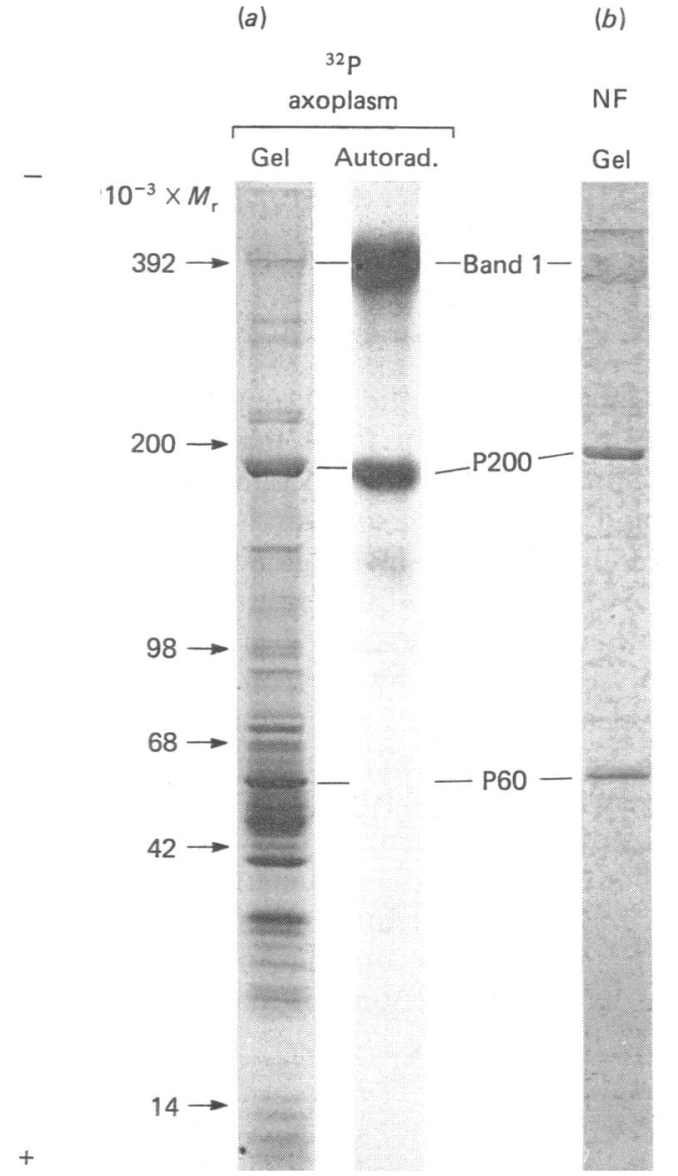

Fig. 1. SDS/polyacrylamide-gel electrophoresis of phosphorylated squid axoplasm (a) and purified neurofilaments (b)

Axoplasm was phosphorylated with $\left[{ }^{32} \mathrm{P}\right] \mathrm{P}_{\mathrm{i}}$ as described in the Methods section and neurofilaments were purified as described by Wais-Steider et al. (1983). Samples were analysed by SDS/polyacrylamide-gel electrophoresis. The gel and corresponding autoradiograph of $\left.{ }^{32} \mathrm{P}\right]$ phosphorylated axoplasm ( ${ }^{32} \mathrm{P}$ axoplasm) are shown in $(a)$, and the gel of purified neurofilaments (NF) is shown in (b). A number of minor polypeptides, the identities of which are unclear, are present in the purified preparation. The polyacrylamide gradient is $3-15 \%$ in $(a)$, and $5-15 \%$ in $(b)$. The mobilities of the $M_{\mathrm{r}}$ marker proteins (see the Methods section) are shown on the left, and the positions of the neurofilament polypeptides are indicated on all lanes.

Transfer of proteins to the nitrocellullose was achieved by applying a heavy weight for about $24 \mathrm{~h}$ in a moist atmosphere (this procedure is termed 'pressure blotting'). Transferred protein was detected by staining the nitrocellulose with $0.5 \%$ Amido Black in $50 \%$ (v/v) methanol $/ 10 \%$ (v/v) acetic acid, and destaining with $50 \%$ (v/v) methanol $/ 10 \%$ (v/v) acetic acid. For antibody staining, the nitrocellulose membrane was first blocked with $2 \%(\mathrm{w} / \mathrm{v})$ bovine serum albumin in phosphatebuffered saline for $1 \mathrm{~h}$, then stained with the first antibody (undiluted tissue-culture supernatant, containing $0.1 \%$ $\mathrm{NaN}_{3}$, from a cell line expressing Anti-IFA) for $1 \mathrm{~h}$, rinsed with phosphate-buffered saline, and then stained with the second antibody (rabbit anti-[mouse IgG $(\mathrm{H}+\mathrm{L})$ ] antibody-horseradish peroxidase conjugate (Miles Scientific), 1/1000 dilution in phosphate-buffered 


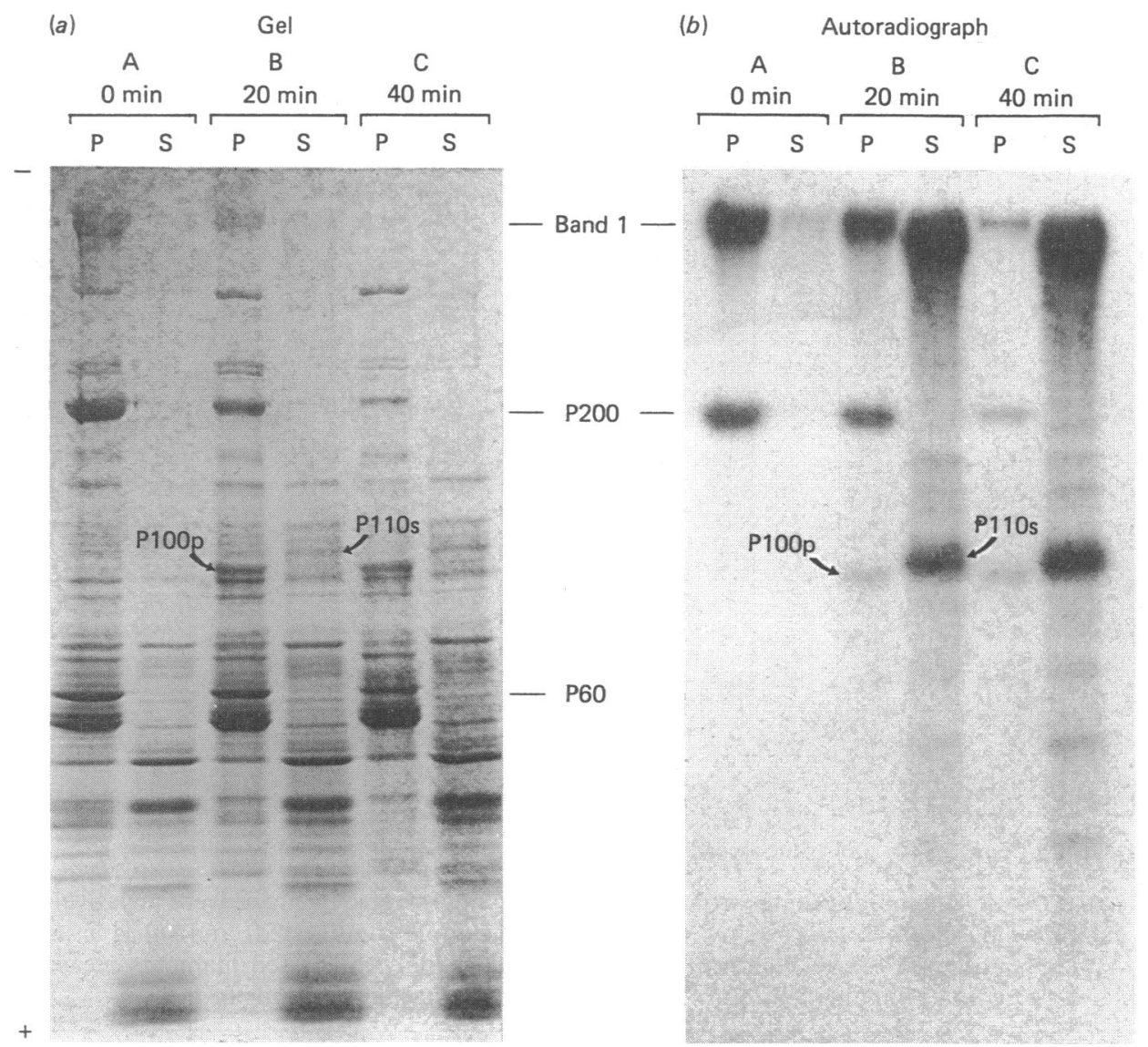

Fig. 2. SDS/polyacrylamide-gel electrophoresis of $\left[{ }^{32} \mathrm{P} \mid\right.$ phosphorylated squid axoplasm after $\mathrm{Ca}^{2+}$-dependent proteolysis

Axoplasm was phosphorylated with [ $\left.{ }^{32} \mathrm{P}\right] \mathrm{P}_{\mathrm{i}}$ and then digested as described in the Methods section. Proteolysis was terminated at time intervals from 10 to $40 \mathrm{~min}$. For the control $(0 \mathrm{~min})$, tosyl-lysylychloromethane was added before addition of $\mathrm{Ca}^{2+}$. Axoplasm was then homogenized and separated into pellet $(\mathrm{P})$ and supernatant $(\mathrm{S})$ fractions by centrifugation. Pellet and supernatant samples incubated for 0,20 and $40 \mathrm{~min}$ (A, B and C respectively) were analysed by SDS/polyacrylamide-gel electrophoresis. The Coomassie Blue-stained gel $(a)$ and corresponding autoradiograph $(b)$ are shown. Comparison by densitometry of the pellet fractions in samples $\mathrm{A}$ and $\mathrm{C}$ from gel $(a)$ shows that, after 40 min proteolysis, $90 \%$ of Band 1 and $85 \%$ of $\mathrm{P} 200$ have been cleaved, but that $60 \%$ of P60 still remains intact. Electron micrographs of filaments from samples A and $\mathrm{C}$ are shown in Fig. 3.

saline containing $0.5 \%$ bovine serum albumin\} for $1 \mathrm{~h}$. The blot was finally rinsed in phosphate-buffered saline and the peroxidase detected by using 3-amino9-ethylcarbazole (Kaplow, 1974).

\section{Electron microscopy}

Samples on carbon support films were negatively stained with $1 \%(\mathrm{w} / \mathrm{v})$ uranyl acetate and examined in a Philips EM200 or EM300 electron microscope at an accelerating voltage of $80 \mathrm{kV}$. Filament widths were measured from the negatives with a Mitutoyo Optical comparator.

\section{RESULTS}

\section{Polypeptide composition of squid neurofilaments}

Fig. 1(b) shows an SDS/polyacrylamide-gel electrophoretogram of purified squid neurofilaments. Two major polypeptides of apparent $M_{\mathrm{r}}$ about 60000 (P60) and 200000 (P200), and a high- $M_{\mathrm{r}}$ component (Band 1), co-purify. We have adopted the nomenclature of Lasek et al. (1979) for the high- $M_{\mathrm{r}}$ component because of uncertainty concerning its exact nature and size. In our system, Band 1 migrates as a diffuse band that stains only faintly with Coomassie Blue. On $3-15 \%$ polyacrylamide gels, with the buffer system of Laemmli (1970), it approximately co-migrates with the glutaraldehyde-crosslinked tetramer of phosphorylase $a$, indicating an $M_{\mathrm{r}}$ of about 400000.

Dephosphorylation of squid axoplasm results in an increase in the mobility during SDS/polyacrylamide-gel electrophoresis of both Band 1 and P200. These components are therefore likely to be highly phosphorylated, and their mobilities, in consequence, are a function of their phosphorylation state (Julien \& Mushynski, 1982; Carden et al., 1985). The apparent $M_{\mathrm{r}}$ values quoted here are thus overestimates (Kaufmann et al., 1984).

\section{$\mathrm{Ca}^{2+}$-dependent proteolysis of $\left[{ }^{32} \mathrm{P}\right]$ phosphorylated axoplasm}

When axoplasm is incubated in $\left[{ }^{32} \mathrm{P}\right] \mathrm{P}_{\mathrm{i}} / \mathrm{KGlu} /$ sucrose buffer the main [ $\left.{ }^{32} \mathrm{P}\right]$ phosphorylated components are P200 and Band 1 (Fig. 1a). Experiments on phosphorylation in intact axons, with $\left[{ }^{32} \mathrm{P}\right] \mathrm{P}_{i}$, indicate that this process is inhibited by $\mathrm{CN}^{-}$, and therefore most 
(a) Control $(0 \mathrm{~min})$

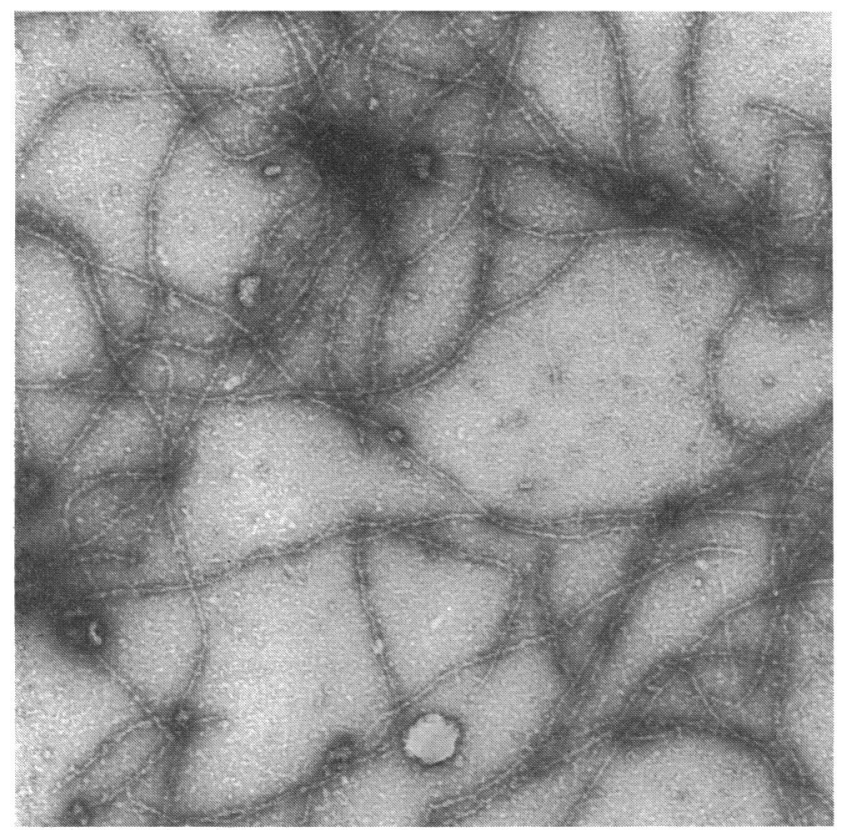

(b) Proteolysed (40 $\mathrm{min})$

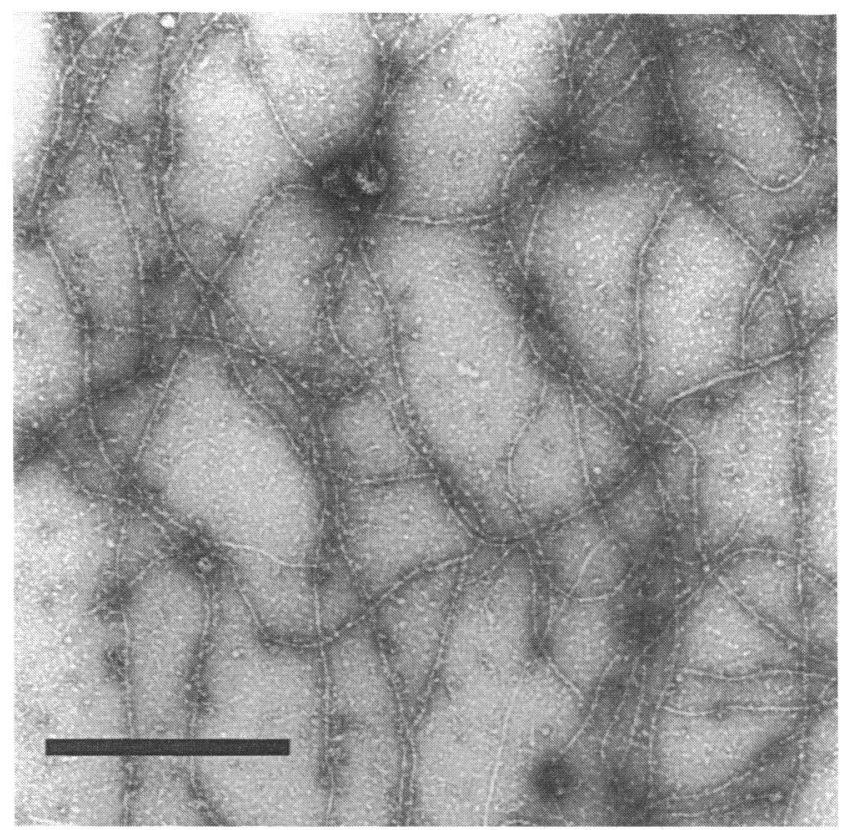

Fig. 3. Electron micrographs of control (a) and proteolysed (b) squid neurofilaments

Before centrifugation, samples of the homogenates from the proteolysis time course in Fig. 2 were negatively stained with $1 \%$ uranyl acetate and examined by electron microscopy. $(a)$ is a control sample (undigested) and $(b)$ is a sample after 40 min digestion. Control filaments measured $7.7 \pm 0.98 \mathrm{~nm}(n=94)$ in diameter and proteolysed filaments measured $7.1 \pm 0.98 \mathrm{~nm}$ $(n=77)$. Micrographs were taken at a magnification of $23000 \times$. The scale bar corresponds to $0.5 \mu \mathrm{m}$.

probably involves the initial incorporation of $\left[{ }^{32} \mathrm{P}\right] \mathrm{P}_{\mathrm{i}}$ into ATP by endogenous mitochondrial oxidative phosphorylation. No degradation was detected after overnight incubation of axoplasm in $\mathrm{KGlu}$ /sucrose buffer, and activation of the proteinase was found to require $>0.5 \mathrm{~mm}$ ionized $\mathrm{Ca}^{2+}$.

Fig. 2 shows the results of a typical proteolysis time course in which $\left[{ }^{32} \mathrm{P}\right]$ phosphorylated neurofilaments were proteolysed for various times. The samples were then separated into pellet and supernatant fractions by centrifugation and analysed by SDS/polyacrylamide-gel electrophoresis. Inspection of lane A shows that all three neurofilament components are present almost exclusively in the pellet fraction, indicating that filaments are stable under the conditions used. Activation of the proteinase results in preferential cleavage of P200 and Band 1, liberating two major phosphorylated fragments into the supernatant, one with an apparent $M_{\mathrm{r}}$ of 110000 , called P110s, and the other a high- $M_{\mathrm{r}}$ fragment. Both fragments stain faintly with Coomassie Blue but are prominent on the autoradiograph. In addition, a fragment of $M_{\mathrm{r}} 100000$, called P100p, appears in the pellet fraction. Inspection of the autoradiograph shows that this fragment, in contrast, contains little of the phosphate label.

Since the soluble high- $M_{\mathrm{r}}$ fragment is highly phosphorylated and has an $M_{\mathrm{r}}$ greater than that of P200, we suggest that it is derived from Band 1 , and that it represents the phosphorylated domain, and a major portion, of this component. The 'ladder-like' pattern of phosphorylated polypeptide fragments in the supernatant appears to result from degradation of this soluble high- $M_{\mathrm{r}}$ fragment, since the appearance of this pattern parallels the progressive broadening of this fragment towards lower $M_{\mathrm{r}}$ as digestion proceeds. In contrast, P110s appears to be relatively resistant to proteolysis, even after more prolonged incubations.

\section{Electron microscopy}

Electron microscopy was used to assess the integrity of filaments after proteolysis. Fig. 3(b) shows a sample of filaments, after 40 min digestion, from the proteolysis time course in Fig. 2. Intact filaments remain, even though SDS/polyacrylamide-gel electrophoresis (see Fig. 2) shows that most of Band 1 and P200 have been cleaved. Furthermore, a comparison of control and proteolysed filaments (Figs. $3 a$ and $3 b$ ) shows no appreciable difference in diameter, though the edges of digested filaments are less sharply defined.

\section{Immuno-blotting}

The monoclonal antibody anti-IFA was used to probe further the arrangement of the polypeptides in the filament and the origin of the proteolytic fragments. Gel samples from the proteolysis time course described in Fig. 2 were stained with the antibody. The nitrocellulose pressure blots are shown in Fig. 4. It can be seen in the undigested control (A) that Anti-IFA binds to both P200 and P60, confirming the observations made by Pruss $e t$ al. (1981). In addition, minor bands of $M_{\mathrm{r}} 75000$ and $M_{\mathrm{r}}$ 62000 are recognized. The identities of these are unclear, though they may correspond to the polypeptides of similar $M_{\mathrm{r}}$ that are seen to co-purify with neurofilaments (see Fig. 1b). In both control (A) and proteolysed (B and C) samples, the antibody binds only to the pellet fraction. P100p, which co-sediments with filaments, 


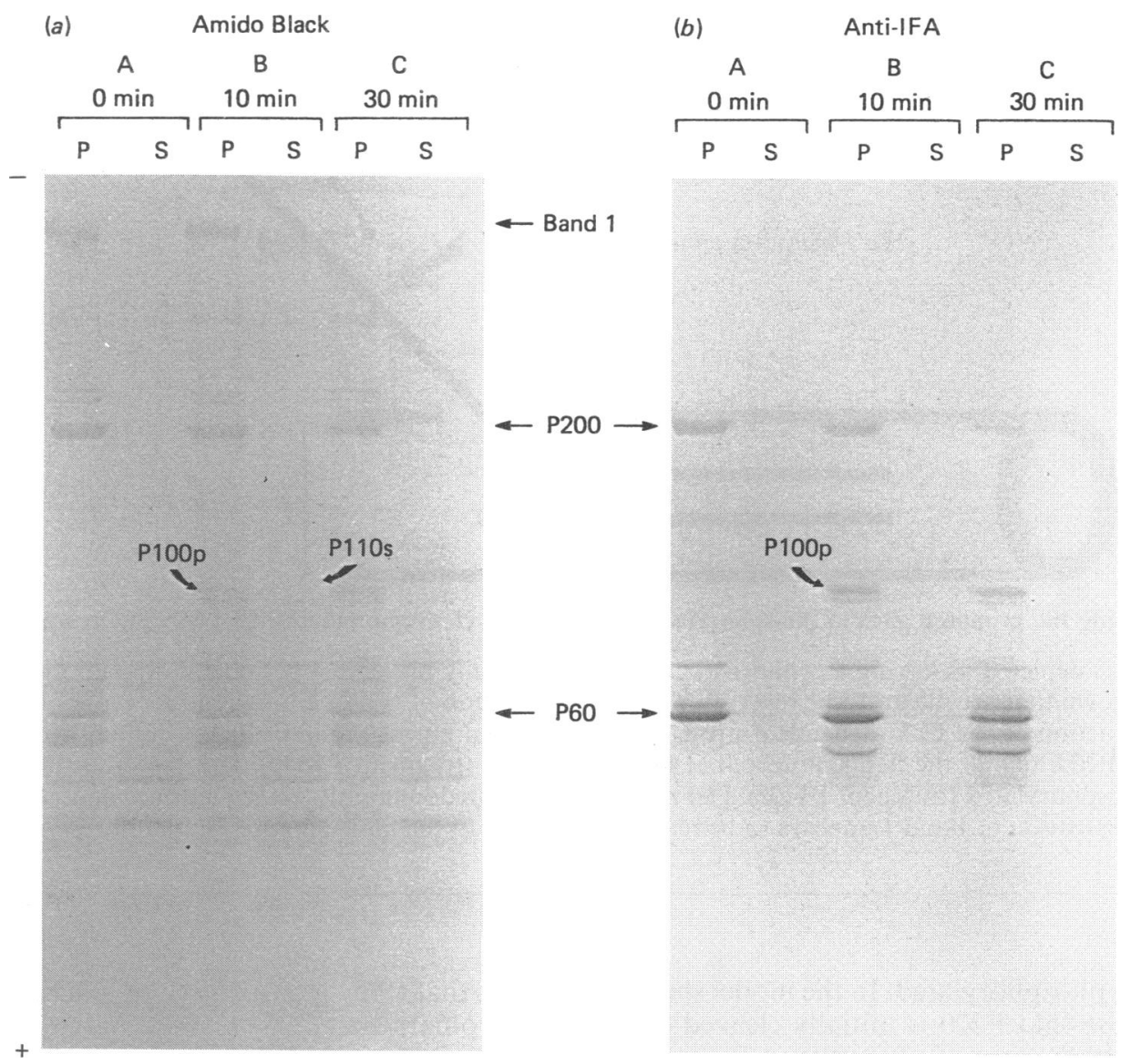

Fig. 4. Anti-IFA immuno-blot of SDS/polyacrylamide-gel electrophoretograms of proteolysed squid axoplasm

Pellet (P) and supernatant (S) samples, corresponding to digestion times of 0,10 and 30 min (A, B and C respectively) from the same proteolysis time course as described in Fig. 2, were analysed by SDS/polyacrylamide-gel electrophoresis and transferred on to nitrocellulose membrane by pressure blotting. Two blots were obtained, one from each side of the gel. One blot (a) was stained with Amido Black in order to detect the transferred protein and the other blot $(b)$ was stained with the antibody Anti-IFA. A number of degradation products of $M_{\mathrm{r}}<60000$ that are not apparent with Amido Black staining of the blot $(a)$, nor with Coomassie Blue staining of the original gel after blotting (results not shown), are detectable in the pellet fractions of digested samples (B and $\mathbf{C}$ ). The origin of these fragments is unclear. Control experiments in which the Anti-IFA incubation was omitted showed no staining of squid axoplasm blots.

binds the antibody, whereas no reaction is seen with the soluble fragments. Since Anti-IFA binds to P200 and not to Band 1, we suggest that P100p is derived from P200. Anti-IFA also binds to a number of other fragments in the pellet fractions of proteolysed samples. We cannot at present distinguish whether the fragments of $M_{\mathrm{r}}$ $<60000$ are derived from P200 or from P60, but they are probably analogous to the highly $\alpha$-helical core fragments identified by proteolysis of Myxicola and vertebrate filaments (see the Discussion section). The two minor Anti-IFA-binding fragments in the $100000-M_{\mathrm{r}}$ region of the blot are probably related to $\mathrm{P} 100 \mathrm{p}$, possibly being produced by cleavage at adjacent sites in P200.

\section{DISCUSSION}

We have studied the enzymic modification of neurofilaments by the endogenous $\mathrm{Ca}^{2+}$-dependent proteinase and protein kinase activities in intact axoplasm. Of the three squid neurofilament polypeptides, only Band 1 and P200 are phosphorylated under the conditions used. The proteinase preferentially cleaves
P200 and Band 1, librating phosphorylated domains, but this does not affect the structural integrity of the filament core.

Immuno-blotting with Anti-IFA shows that P100p is derived from $\mathrm{P} 200$. Thus, during proteolysis, one would expect the formation of a fragment with an $M_{\mathrm{r}}$ of about 100000 that would correspond to the remaining part of P200 and contain the bulk of the phosphate label that was originally present on the undigested component. A strong candidate for this fragment is P110s. However, at present we do not discount the possibility that a proportion of this band might be derived by digestion of the soluble, highly phosphorylated, high- $M_{\mathrm{r}}$ fragment of Band 1. The poor staining of Band 1 with Coomassie Blue and the uncertainty concerning its nature and exact size preclude an unambiguous assignment of the breakdown fragments derived from this component. The resolution of this ambiguity must await further immunochemistry.

We suggest that proteolysis defines two distinct filament regions for the squid neurofilament: a core that forms the backbone of the filament and a peripheral 


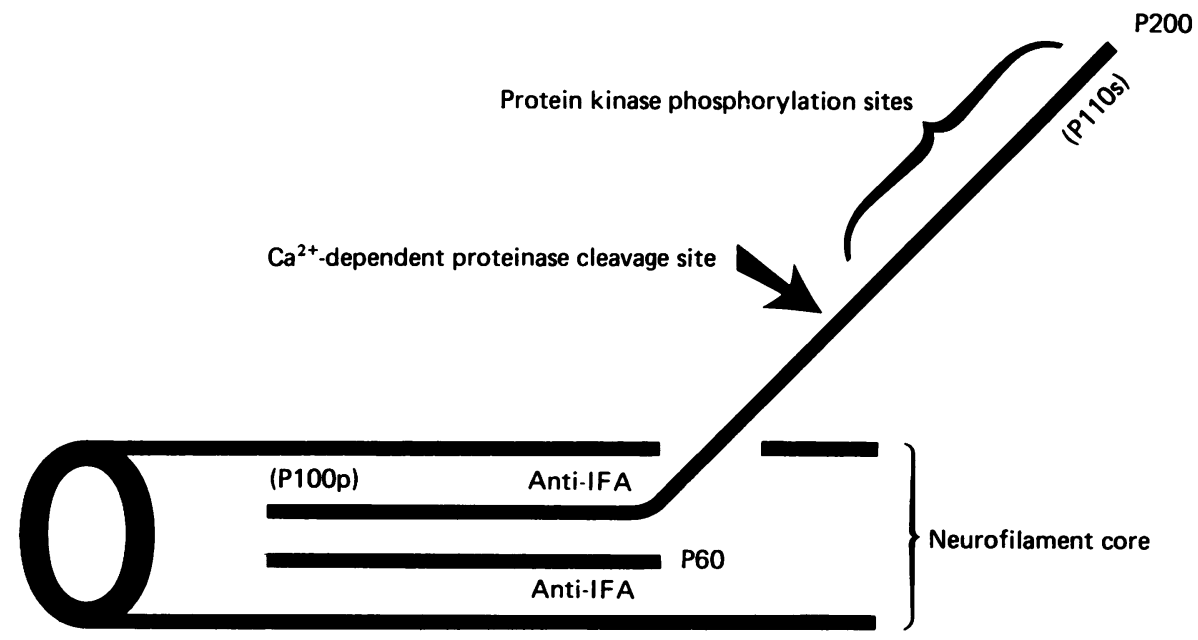

Fig. 5. Diagram showing the proposed sites of phosphorylation, proteolytic cleavage and anti-IFA binding in squid neurofilaments

The neurofilament is depicted as having a cylindrical core with a laterally projecting peripheral domain. Single P200 and P60 polypeptides are shown that are meant to be representative of the location of these components throughout the neurofilament. The major sites of action of the $\mathrm{Ca}^{2+}$-dependent proteinase and protein kinase are indicated. Limited proteolysis defines two major domains in P200, one in the filament core that binds Anti-IFA (fragment P100p), and the other located peripheral to the core that is phosphorylated (fragment P110s). P60 resides at least predominantly within the core and is resistant to limited proteolysis. The disposition of Band 1 appears to be mainly peripheral to the filament core, but its precise nature and location are unclear.

region that is highly phosphorylated. In the model shown in Fig. 5, we propose that P200 is initially cleaved at a single highly sensitive site to generate two major fragments. One fragment, $P 100 \mathrm{p}$, sediments with intact filaments, and functions to anchor the intact polypeptide in the core. The other, P100s, is released without disrupting the filament structure, suggesting that it is situated peripheral to the filament core. Most of the phosphate on P200 is located on this fragment. Band 1 is cleaved to produce a soluble, highly phosphorylated, fragment, which represents a major portion of the undigested component. Band 1 therefore appears to be localized predominantly peripheral to the filament core. In contrast, P60, which is not phosphorylated, is relatively resistant to limited proteolysis, suggesting that it is localized largely within the core.

In spite of great differences in both the number and size of the component polypeptides, results indicating peripheral and core regions have also been obtained for neurofilaments from the annelid Myxicola (Eagles et al., $1981 a, b$ ) and from vertebrates (Willard \& Simon, 1981; Chin et al., 1983; Julien \& Mushynski, 1983; Geisler et al., 1983). Furthermore, a comparison of the available amino acid sequences for vertebrate neurofilaments indicates homology between the core regions of the triplet polypeptides (Geisler et al., 1985a). A useful probe for this conserved sequence is the monoclonal antibody Anti-IFA, which recognizes all classes of intermediate filament (Pruss et al., 1981), and binds to the core regions of neurofilament polypeptides from both vertebrates (Geisler et al., 1983) and Myxicola (Eagles \& Maggs, 1985). In digested samples of squid neurofilaments, Anti-IFA binds to P60 and to P100p, which sediment with intact filaments, but not to P110s, nor to the high- $M_{\mathrm{r}}$ fragment, which are both soluble. Thus we suggest that P60 and P100p are probably analogous to the core regions defined for other intermediate filaments and that they constitute the backbone of the squid neurofilament.

The relationship between the three squid neurofilament polypeptides is unclear. Peptide mapping with papain reveals homology between Band 1 and P200, and some similarity between P200 and P60 (Eagles et al., 1980). On this basis it was suggested that Band 1 might represent a cross-linked complex of two or more $P 200$ polypeptides. Certainly, we would expect P200 and P60 to show homology, since immunoblotting demonstrates that they both contain the Anti-IFA epitope. However, Anti-IFA does not recognize Band 1 on nitrocellulose pressure blots, though Amido Black staining confirms that Band 1 is transferred (Fig. 4a). Nevertheless, it seems clear that Band 1 is an integral component of the neurofilament, since it consistently co-purifies with P60 and P200, and all three components exhibit identical solubility and reassembly properties over a wide range of $\mathrm{pH}$ and ionic strength (A. Brown \& P. A. M. Eagles, unpublished work).

Thus the results presented here show that, despite a unique polypeptide composition, neurofilaments from squid appear to conform to similar structural principles as those found for other phyla. The evolutionary conservation of the intermediate-filament sequence in the core region (Geisler et al., 1982, 1985a) presumably reflects the structural constraints imposed by a requirement for assembly into a filament. In contrast with this core, the highly phosphorylated $C$-terminal extension (Julien $\&$ Mushynski, 1983) is a unique feature of neurofilaments, and, as such, presumably represents specialization for a function peculiar to neuronal processes. We consider, however, that the wide variation in sequence and size of the $C$-terminal peripheral regions (Geisler et al., 1983, $1985 b$; Phillips et al., 1983), rather than reflecting distinct species-specific functional requirements, is probably due to less specific structural and functional 
constraints for these domains than for the core. Since these regions are peripheral to the filament backbone and are not essential for filament integrity, they have been considered likely candidates for the cross-bridges that appear to interconnect neurofilaments in axoplasm (Hirokawa et al., 1984), and they may also provide a substrate for the action and interaction of other more dynamic cytoskeletal components.

\section{Note added in proof (received 31 July 1986)}

Broadly similar results to those presented here have recently been reported by Gallant $e t$ al. (1986).

We thank the Director and staff of the Laboratory of the Marine Biological Association at Plymouth for the supply of squid and laboratory facilities. The anti-IFA cell line was generously given by Dr. Rose Yasin. A. B. was supported by a Medical Research Council Research Studentship.

\section{REFERENCES}

Carden, M. J., Schlaepfer, W. W. \& Lee, V. M.-Y. (1985) J. Biol. Chem. 260, 9805-9817

Chin, T.-K., Eagles, P. A. M. \& Maggs, A. (1983) Biochem. J. 215, 239-252

Eagles, P. A. M. \& Maggs, A. (1985) Ann. N.Y. Acad. Sci., 455 779-781

Eagles, P. A. M., Gilbert, D. S. \& Maggs, A. (1980) Biochem Soc. Trans. 8, 484-487

Eagles, P. A. M., Gilbert, D. S. \& Maggs, A. (1981a) Biochem. J. 199, 89-100

Eagles, P. A. M., Gilbert, D. S. \& Maggs, A. (1981b) Biochem. J. 199, 101-111

Gallant, P. E., Pant, H. C., Pruss, R. M. \& Gainer, H. (1986) J. Neurochem. 46, 1573-1581
Geisler, N., Plessmann, U. \& Weber, K. (1982) Nature (London) 296, 448-450

Geisler, N., Kaufmann, G., Fischer, S., Plessmann, U. \& Weber, K. (1983) EMBO J. 2, 1295-1302

Geisler, N., Plessmann, U. \& Weber, K. (1985a) FEBS Lett. 182, 475-478

Geisler, N., Fischer, S., Vandekerckhove, J., Van Damme, J., Plessmann, U. \& Weber, K. (1985b) EMBO J. 4, 57-63

Gilbert, D. S. (1976) J. Physiol. (London) 266, 81P-83P

Gilbert, D. S., Newby, B. J. \& Anderton, B. H. (1975) Nature (London) 256, 586-589

Hirokawa, N., Glicksman, M. A. \& Willard, M. B. (1984) J. Cell Biol. 98, 1523-1536

Julien, J.-P. \& Mushynski, W. E. (1982) J. Biol. Chem. 257 , $10467-10470$

Julien, J.-P. \& Mushynski, W. E. (1983) J. Biol. Chem. 258, 4019-4025

Kaplow, L. S. (1974) Am. J. Clin. Pathol. 63, 451

Kaufmann, E., Geisler, N. \& Weber, K. (1984) FEBS Lett. 170, 81-84

Laemmli, U. K. (1970) Nature (London) 227, 680-685

Lasek, R. J., Krishnan, N. \& Kaisermanabramof, T. R. (1979) J. Cell Biol. 82, 336-346

Pant, H. C. \& Gainer, H. (1980) J. Neurobiol. 11, 1-12

Pant, H. C., Shecket, G., Gainer, H. \& Lasek, R. J. (1978) J. Cell Biol. 78, R23-R27

Pant, H. C., Yoshioka, T., Tasaki, I. \& Gainer, H. (1979) Brain Res. 162, 303-314

Phillips, L. L., Autilio-Gambetti, L. \& Lasek, R. J. (1983) Brain Res. 278, 219-223

Pruss, R. M., Mirsky, R., Raff, M. C., Thorpe, R., Dowding, A. J. \& Anderton, B. H. (1981) Cell (Cambridge, Mass.) 27, 419-428

Roslansky, P. F., Cornell-Bell, A., Rice, R. V. \& Adelman W. J., Jr. (1980) Proc. Natl. Acad. Sci. U.S.A. 77, 404-408

Wais-Steider, C., Eagles, P. A. M., Gilbert, D. S. \& Hopkins, J. (1983) J. Mol. Biol. 165, 393-400

Willard, M. \& Simon, C. (1981) J. Cell Biol. 89, 198-205 